\title{
Issues \& Trends in Design and Analysis for better Products
}

\author{
Chandrasekaran K \\ Professor and Dean, \\ R.M.K.Engineering College, Chennai, India
}

\begin{abstract}
The breakneck speed at which computational techniques have evolved, assisted by speedier developments in computer graphics and 3- modeling, the contribution of design and analysis to a faster product development process has been phenomenal in the past decade. All along, the role of finite element analysis has been increasingly acknowledged and accepted as crucial to solutions of engineering and scientific problems. Since all problems that could be mathematically modeled as discrete or continuum models, irrespective of their spatial or temporal complexity, the resulting boundary or initial value problems could be effectively solved with acceptable accuracy through the finite element formulations.
\end{abstract}

Key words: FEA, CAE, Power of Pressure Systems, Verification and validation, Fuzuy Logic, Computational Mechanic

\section{INTRODUCTION}

This has opened up a plethora of their applications in the design and analysis of products/structures which, to simply put it, is mind boggling! This opportunity has been promptly grabbed by software developers in areas related to CAD/CAE to provide highly user-friendly, visually rich, computationally efficient, and reasonably affordable application packages as useful tools to the industry. However, the indiscriminate and many times, inaccurate uses of these packages have blurred their efficacy. There is a feeling that the education and training that ought to have followed the installation of the software have not been effective in ensuring realistic and acceptable solutions to complex problems. This is something to be pondered over. However, it would be eminently wrong to ignore or belittle the relevance of these packages to provide a good, fast and acceptable tool for design and analysis. But what needs to be understood is the positioning of the design and analysis in the product development process, and the need for a good understanding of the fundamentals of FEA. In what follows we discuss some current trends in FEA and the increasing importance of the product development process, of which this forms a part, to the growth of a business. The material below is taken from the findings in the Annual Industry Report of an independent organization, NAFEMS, headquartered in the UK( www.nafems.org), and devoted to establish a community of analysts for closer interaction in the development and applications of Finite Element Analysis.

\section{TRENDS IN DESIGN AND ANALYSIS}

A recent study by NAFEMS mentions that in the last decade or so, finite element technology has become a generic enabling technology across a broad spectrum of industrial sectors. However, in view of the diversity of issues related to different industrial sectors, the scale, depth and level of maturity of application varies widely. Nevertheless there are a number of common issues reported from all industrial sectors. FENET was a four year project funded by the EU and initiated in 2001 to provide a vehicle for informed users of FEA technology in Europe to network together on a regular basis. Its broad objective was to improve the competitiveness of European industry through better use of finite element and related technologies. The Annual Industry Meeting reviewed the state of practice in 8 industry sectors, with emphasis on a defined theme, and attempted to "roadmap" key industry technology issues. These issues are documented in a series of Annual Industry Reports. The major objectives of the study were concerned with:

1. Integration of finite element technology and simulation into the wider business enterprise in order to deliver real business benefit.

2. How to ensure and measure quality of finite element analysis. (this covers diverse topics including verification and validation, Q.A., the role of testing, data provenance etc.)

3. How to categorise and analyse modern materials.

4. How to allow, in a rational way, for the uncertainty that characterises real world problems and there is increased interest in methods such as probabilistic analysis, fuzzy logic and possibility theory.

The NAFEM report also discusses the emerging applications in the various industry sectors, like Consumer Goods, Bio-Medical, Process \& Manufacturing, and Power \& Pressure Systems, aong others. 


\section{CONSUMER GOODS SUMMARY OF FINDINGS}

The consumer Goods Industry Sector is a wide one with products varying from small handheld devices to heavier household equipment. Modern consumer goods have short operational lives compared to many other products; cycle times need to be correspondingly short, with the gap from concept to product being measured in months rather than years. To effectively integrate FE into the design process communication between CAD packages and analysis packages must be easy and accurate, (not necessarily the same thing).

Accurate tracking of material properties that go with the model and validation of the results must all be considered as part of the analysis process that is followed all the way to the display shelf. CAD plug-ins and "designer level" analysis systems promise "one-click" analysis, but the question of the required level of training and expertise for these programs has yet to be properly answered independently from the vendors. With the next generation of these packages promising automatic optimisation of features based on broader criteria than shape alone, the line between draughtsman and analyst is becoming difficult to draw.

Business drivers include reduction time to market. Barriers include high costs of simulation and quality control, low CAD integration and lack of people with adequate skills.

\section{BIOMEDICAL SUMMARY OF FINDINGS}

The use of analysis and simulation for bio-medical purposes is increasing dramatically but is still quite immature. In contrast to other industrial sectors most analysis work is carried out by "specialists" in consultancies, universities or research establishments and industrial "practises" are in there infancy. Nevertheless the potential benefits are substantial.

There are two distinct drivers; firstly an improved understanding of the biomechanics of human body with view to development of artificial implants, e.g. hip replacements, artificial heart valves. Secondly the simulation of body kinematics in crash scenarios in response to the need for safer cars, trains etc.

In the first case the use of CAE techniques gives insight into the load mechanisms, material behaviour and response of the implants and the biomedical materials (bone, cartilage, ligaments, muscles, etc.). They can support the product design and development process in many aspects, such as wear predictions, structural behaviour, component loading etc. In the second area the interest is understanding how the human body interacts in crash situations when subject to very rapid decelerations, with a view to designing safer vehicles.

An additional group of problems stems from the ever increasing use of micromechanisms and microsystems in medical applications. Typical devices include microfluidic, electrical and sensing components. Problems associated with design and use of these devices requires solution to coupled physics issues such as; flow, heat transfer, chemistry and diffusion or structural analysis, electrostatics, electromagnetics and plasma.Advanced tools are emerging. However the computing power required is demanding and there is considerable scope for research directed at better models.

\section{V.PROCESS AND MANUFACTURING SUMMARY OF FINDINGS}

Predictive simulation of manufacturing processes is an area of fast growing interest for industry. Indeed it can be argued that in a number of cases the advantages of simulation, over traditional design approaches are significantly greater in this field, than in areas where FE and numerical simulation are already well-established. Process simulation includes, material forming, such as casting, forging, stamping, injection molding, plastic sheet forming and the like, but also welding and heat treatment simulation, such as surface treatments, quenching, and thermo-mechanical treatments in general. Methods and software products are particularly complex, as problems are highly non-linear, transient, requiring integration of a variety of physical phenomena and process parameters, and often related to variables of different physical fields

Process optimisation, being a business driver from the perspectives of, productivity, cost and time to market however plays a key role. It is typically multi-objective: with a variety of inherently different technical issues, furthermore operational efficiency and cost targets have to be taken into account at the same time. Evolutionary and hybrid approaches are required, as well as MCDM methods to find out the best compromise among the nondominated optimal solutions.All this is at the forefront of numerical simulation. Hence a network of competencies (and related education) is crucial to support industrial applications.

\section{POWER AND PRESSURE SYSTEM SUMMARY OF FINDINGS}

This industry ranges from the relatively demanding safety-challenged nuclear power sector to the manufacturers of industrial pressure vessels. The industry is characterised by codes of practise which seek to embody contemporary experience and which to some extent have yet to be adapted to take account of modern 
CAE capabilities. Thus for example much design is by "rule" although "design by analysis" is being introduced in some codes. In this environment the role for analysis and simulation is the traditional one of post design demonstration of structural integrity. In common with many industries there are demands in various sectors (e.g. nuclear power) to extend the life of existing plant, while at the same time improving operational efficiency. These demands introduce particular challenges in respect of the characterisation of ageing material (where the ageing process can involve corrosion and material degradation, fatigue and irradiation) and how to treat the uncertainties that arise. In some sectors (notably nuclear) the end-oflife decommissioning process can be dominated by safety concerns and simulation plays an important part in developing the associated safety case. Again long term material behaviour and the characterisation of other epistemic uncertainty are important issues. There is considerable interest in probabilistic models in respect of the latter. There is also the important issue of regulatory certification in areas such as the nuclear industry, including the movement of nuclear materials by transport containers and systems.

The business drivers include a need for the design by analysis, compliance with design codes and evaluation of limit loads. Barriers include difficulty with validation of FEA results, terminology, insufficient awareness of the DBA manual and difficulty with incorporation of empirical knowledge into the FEA.

\section{PRODUCT AND SYSTEM OPTIMISATION SUMMARY OF FINDINGS}

This covers the need for simulation technology which allows the "system" to be optimised for a wide range of criteria and conditions. It includes for example improved methods of topology and weight optimisation, methods for treating uncertainties more rationally (e.g. reliability-based design optimisation) in addition to the detailed treatment of non-linear effects such as contact, friction, buckling etc. Other areas of interest are large strain effects encountered in modern forming and production processes and many others, impact modelling (including deformational response with large kinematics).

The quest of all engineering processes is to make things better. In the area of Computational Mechanics there have been huge advances in the last 30 years with parallel developments in computers and computational algorithms. Finite Element Analysis has evolved to such a stage of competency that the engineer/physicist can analyse any defined physical situation, linear or non-linear provided the material properties are known.
In the last ten years there has been significant academic research in the area of Structural Optimization to the stage where the algorithms needed for size, shape, topology and topography optimization are becoming more reliable and robust. We are now starting to see some limited commercial uptake of these analytical optimizers replacing the traditional engineering intuitively/heuristic driven iterative design optimization methods.

Equally as important, but still significantly lacking, is the integration of manufacturing process models into the design optimization loop. Indeed if we are to be commercially serious for the product under consideration then we should also include financial, marketing, environmental, support and service and retirement into the design optimization. Each of these activities has different analysis processes and data structures and responsibility resides in different locations in any commercial organization. Even between analysis and manufacturing models there are significant integration problems. This gap becomes even greater when other commercial processes are involved. The challenge is therefore to guide the development and uptake of these new integrated analytical processes into true design optimization and to provide direction to all parties involved; code developers, researchers, designers and manufactures as to how the Computational Mechanics community should proceed from here.

\section{CONCLUSION}

The material given above forms the basic findings of the study reported and are as much relevant to Indian industries as they are to the EU. In delivering an effective education and training in these important areas of design and analysis, the role of the academia, R\&D organizations and the Software Developers becomes very crucial. There is a need to bring all the stake-holders together in an umbrella organization which would promote a focused education and training with a clear skill development and understanding of the fundamentals. Educational institutions should not shy away from the required investments in the software packages. At the same time, the vendors have a responsibility in the supply of packages at economical and affordable rates. The R\&D organizations have a big role in providing the right exposure to practical applications by encouraging students to undertake projects that have a practical significance, and by taking part in the educational process. 


\section{REFERENCES}

1. N.Lerke \& D.Ellis, May 2005, "A Review of FEA Technology Issues Confronting the Consumer Good Industry", Summary of Project Findings, Proceedings of FENET Meeting, Malta, pp.79-85

2. Laurence Marks, May 2005, "A Review of FEA Technology Issues Confronting the Bio-Mechanics Industry", Summary of Project Findings, Proceedings of FENET Meeting, Malta, pp.105-110.
3. Gerrit-Jan Dop, May 2005, " A Review of FEA Technology Issues Confronting the a Manufacturing Industry", Summary of Project Findings, Proceedings of FENET Meeting, Malta, pp.39-46

4. Nawal K.Prinja and lain Davidson, May 2005, " A Review FEA Technology Issues Confronting the Power Industry", Summary of Project Findings, Proceedings of FENET Meeting, Malta, pp.95-103 